\title{
SCHRIFTEN DER VEREINIGUNG ÖSTERREICHISCHER BIBLIOTHEKARINNEN UND BIBLIOTHEKARE (VÖB), BAND 1-14
}

Herausgeber im Auftrag der VÖB: Dr. Harald Weigel

Band 1: Eveline Pipp (Hrsg.): Zugang zum Fachwissen. ODOK '05 ; 11. Österreichisches Online-Informationstreffen, 12. Österreichischer Dokumentartag ; 13.-16. September 2005, Freie Universität Bozen. Graz : Feldkirch : Neugebauer, 2007. ISBN: 9783853762813, 220 Seiten, Broschur, EUR 39,90.-

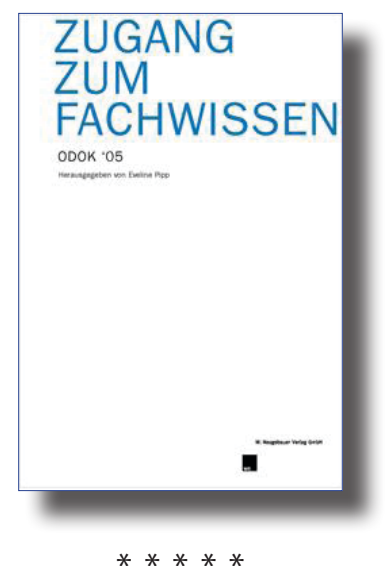

Band 2: Harald Weigel (Hrsg.): Wa(h)re Information. 29. Österreichischer Bibliothekartag Bregenz 2006. Graz : Feldkirch : Neugebauer, 2007. ISBN: 9783853762820, 317 Seiten, Broschur, EUR 39,90.-

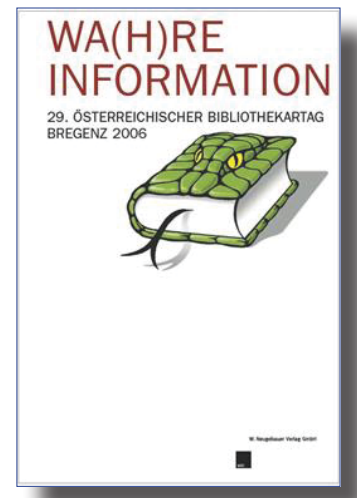


Band 3: Harald Tersch: Schreibkalender und Schreibkultur. Zur Rezeptionsgeschichte eines frühen Massenmediums. Graz : Feldkirch : Neugebauer, 2008. ISBN: 9783853762837, 120 Seiten, Broschur, EUR 24,80.-

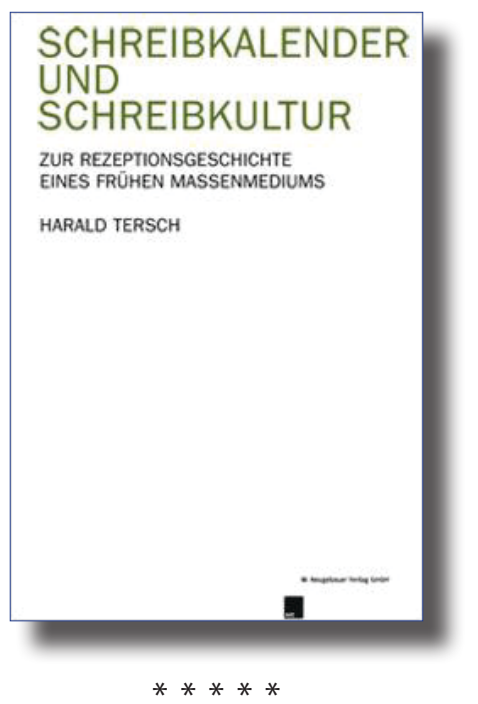

Band 4: Marion Kaufer: Erwerbungsprofile in wissenschaftlichen Bibliotheken. Eine Bestandsaufnahme. Graz : Feldkirch : Neugebauer, 2008. ISBN: 9783853762844, 91 Seiten, Broschur, EUR 22,00.-

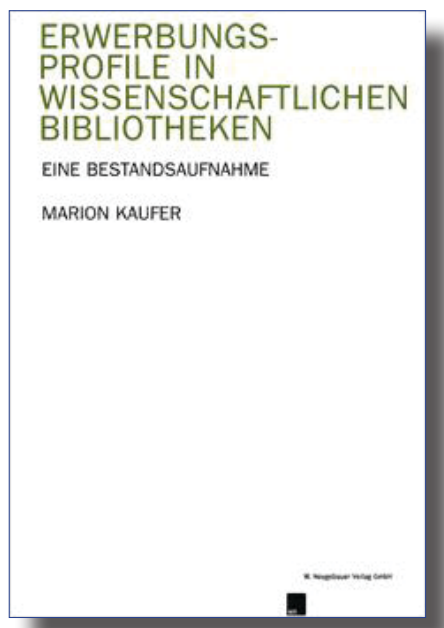


Band 5: Eveline Pipp (Hrsg.): Informationskonzepte für die Zukunft. ODOK '07. Graz : Feldkirch : Neugebauer, 2008.

ISBN: 9783853762851, 204 Seiten, Broschur, EUR 39,90.-

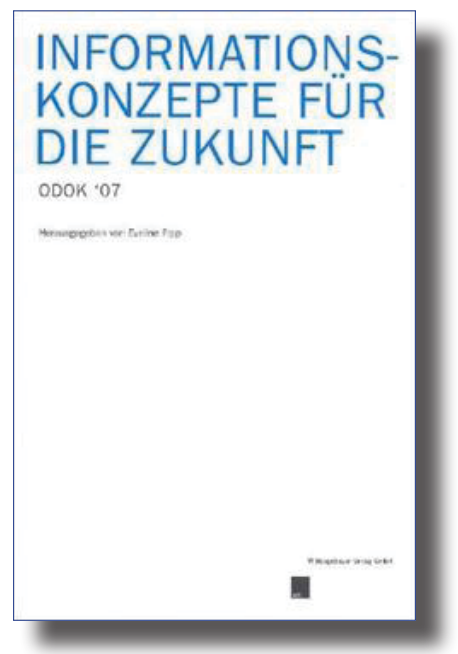

$* * * * *$

Band 6: Michael Katzmayr: Aufteilung des Erwerbungsbudgets und der Erwerbungskosten in Universitätsbibliotheken. Prinzipien wirtschaftlichen Handelns im Bestandsaufbau. Graz : Feldkirch : Neugebauer, 2009.

ISBN: 9783853762868, 80 Seiten, Broschur, EUR 18,00.-

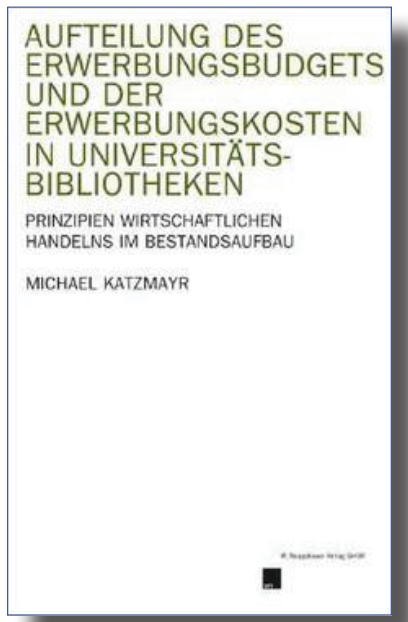


Band 7: Ute Bergner; Erhard Göbel (Hrsg.): The Ne(x)t Generation - Das Angebot der Bibliotheken. 30. Österreichischer Bibliothekartag Graz 2009. Graz: Feldkirch : Neugebauer, 2010. ISBN: 9783853762875, 372 Seiten, Broschur, EUR 39,90.Vergriffen/Print-on-Demand: auf Anfrage

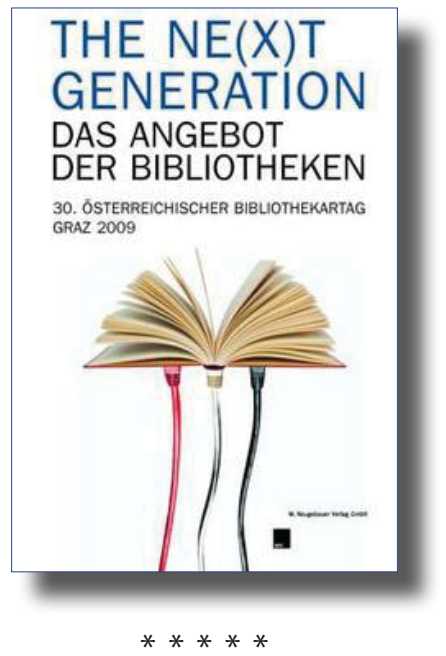

Band 8: Norbert Schnetzer (Hrsg.): Trends, Megatrends, Sackgassen. Die Sondersammlungen im 21. Jahrhundert. Festkolloquium für Dr. Hans Zotter im Rahmen des 30. Österreichischen Bibliothekartages „The Ne(x)t Generation - Das Angebot der Bibliotheken". Graz : Feldkirch : Neugebauer, 2010. ISBN: 9783853762882, 115 Seiten, Broschur, EUR 24,80.-

\section{TRENDS, MEGATRENDS, SACKGASSEN}

DIE SONDERSAMMLUNGEN

IM 21. JAHRHUNDERT 
Band 9: Schaffner, Verena: FRBR in MAB2 und Primo - ein kafkaesker Prozess? Möglichkeiten der FRBRisierung von MAB2-Datensätzen in Primo, exemplarisch dargestellt an Datensätzen zu Franz Kafkas „Der Process“. Graz : Feldkirch : Neugebauer, 2012.

ISBN: 9783853762899, 104 Seiten, Broschur, EUR 22,00.-

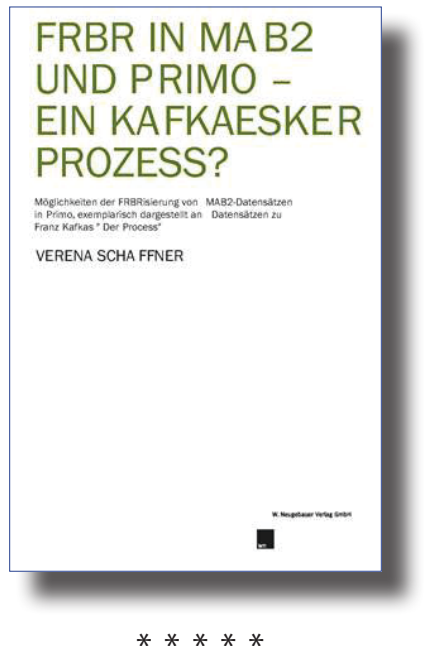

Band 10: Bauer, Bruno, Christina Köstner-Pemsel und Markus Stumpf (Hrsg.): NS-Provenienzforschung an österreichischen Bibliotheken: Anspruch und Wirklichkeit. Graz : Feldkirch : Neugebauer, 2011.

ISBN: 9783853762905, 542 Seiten, Broschur/Fadenheftung, EUR 59,90.-

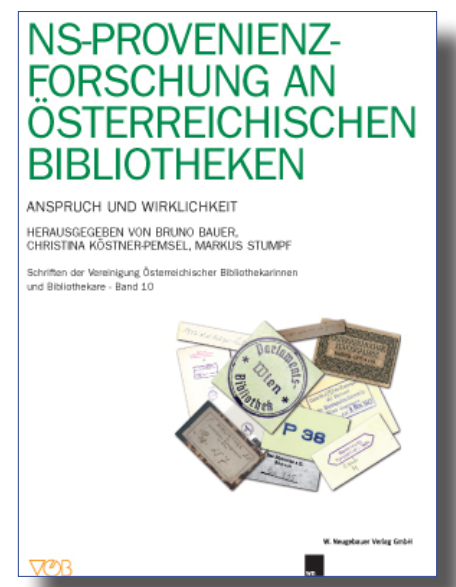


Band 11: Niedermair, Klaus (Hrsg.): Die neue Bibliothek. Anspruch und Wirklichkeit. 31. Österreichischer Bibliothekartag, Innsbruck 2011. Graz : Feldkirch : Neugebauer, 2012.

ISBN: 9783853762912, 448 Seiten, Broschur, EUR 39,90.-

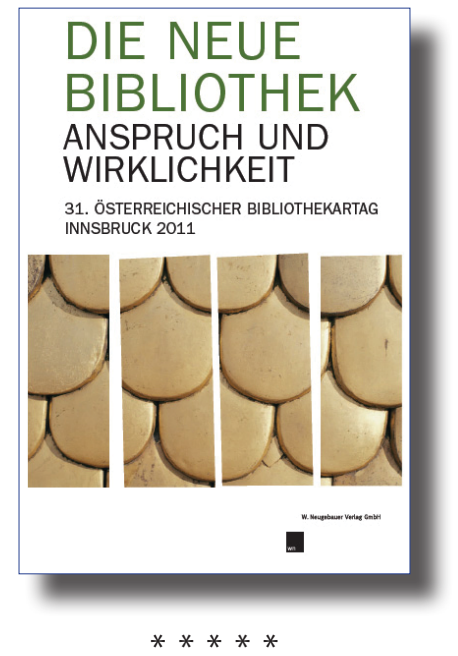

Band 12: Hensel, André: FaMI und ABID-Assistenz: quo vaditis? Eine Vergleichsstudie zur beruflichen Aus-, Fort- und Weiterbildung auf der mittleren Qualifikationsebene im Archiv-, Bibliotheks-, Informations- und Dokumentationswesen (ABID) des deutschsprachigen Raumes. Graz : Feldkirch : Neugebauer, 2012. ISBN: 9783853762929, 158 Seiten, Broschur, EUR 23,50.-

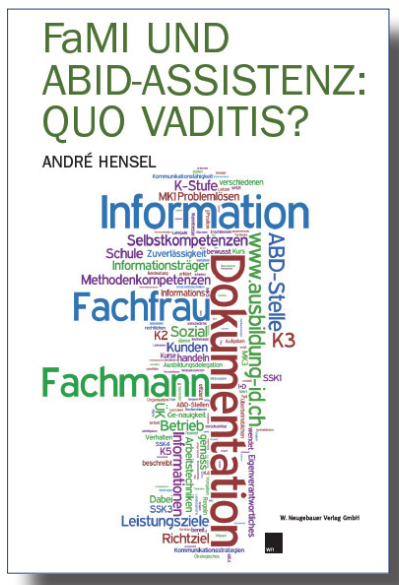


Band 13: Bauer, Bruno; Gumpenberger, Christian; Schiller, Robert (Hrsg.): Universitätsbibliotheken im Fokus. Aufgaben und Perspektiven der Universitätsbibliotheken an öffentlichen Universitäten in Österreich. Graz : Feldkirch : Neugebauer, 2013. ISBN: 9783853762936, 418 Seiten, Broschur, EUR 52,00.Vergriffen/Print-on-Demand: auf Anfrage

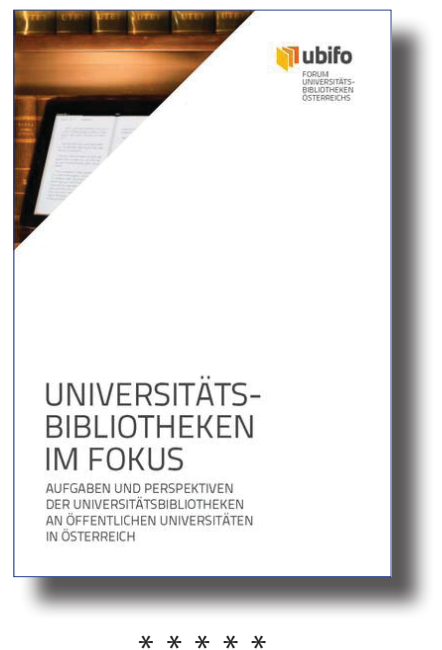

Band 14: Bauer, Bruno; Ferus, Andreas; Pauser, Josef (Hrsg.): Offen(siv)e Bibliotheken: Neue Zugänge, neue Strukturen, neue Chancen, 32. Österreichischer Bibliothekartag in Wien 2015. Graz : Feldkirch : Neugebauer, 2016. ISBN: 9783853762943, 229 Seiten, Broschur, EUR 48,00.-

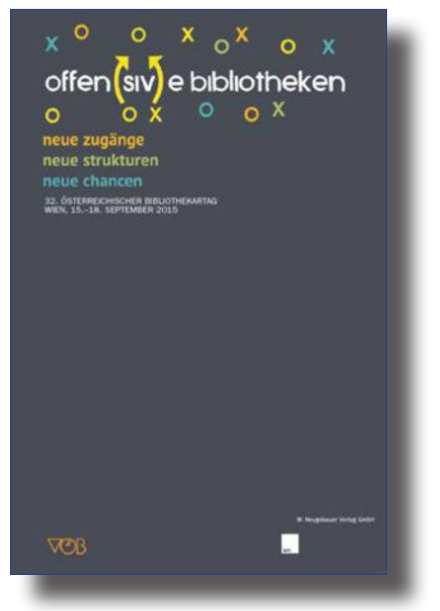




\section{Bestellungen an:}

Wolfgang Neugebauer Verlag, Auslieferung

Mühleweg 134, 6822 Düns

Österreich

Tel./Fax: +43 55245199

E-Mail: info@wnverlag.com

Lieferung zzgl. Versandspesen. 